\title{
Peculiarities of thermal inspection of materials with short observation time
}

\author{
by VAVILOV V.P.*, GRINZATO E.\#, BISON P.G.\# and MARINETTI S.\# \\ ^Tomsk Polytechnic University, Russia, 634028, Tomsk, 28, Savinykh, 3 \\ \#ITEF-CNR, Corso Stati Uniti, 4, 35020-Padova, Italy

\begin{abstract}
Modem thermal NDT tends to deal with millicentigrade temperature signals occurring for tens of milliseconds. This concerns inspection of either high-conductive materials, such as steel, aluminium etc., or thin materials where heat processes are fast. The paper highlights the correlations between heat pulse duration, life time for signal over defect and frame time of an IR imager.
\end{abstract}

\section{Introduction}

Appearance of powerful short-pulse optical heaters and high-speed IR imagers has ignited new applications of the thermal NDT (TNDT) where: 1 ) frame time of an IR imager is $30-40 \mathrm{~ms}$ (shorter time is possible with loss of lines number; also quasi instantaneous thermal images could be obtained with focal plane array imagers); 2) heat pulse is delivered for 2-10 ms, i.e. within frame time of common IR imagers; 3 ) defect signals exist for tens or hundreds of millisecond; 4 ) operator is out of operation due to its slow reaction time (about $0.1 \mathrm{~s}$ ). These TNDT features are especially important in the inspection of carbon epoxy plastics, carboncarbon composites, plasma sprayed coatings, corroded steel and aluminium parts.

\section{Fast thermal processes (hyperbolic equation of heat conduction)}

With the term "fasi thermal process" we specify the cases where build-up time for defect signal competes the technical limits of apparatus and is shorter than operator reaction time.

Principle limit to the fastest thermal process is due to finite heat velocity, i.e. velocity of ballistic phonons. In solids this value is about some kilometres per second. Experimental studies of phonons phenomena have been done for pure crystals at low temperatures [1]. Recently the technique for 2D imaging of phonons has become available [2].

Heat transfer with finite heat velocity is governed by the hyperbolic equation:

$$
\frac{\partial T}{\partial \tau}+\tau_{r} \frac{\partial^{2} T}{\partial \tau^{2}}=\alpha \nabla^{2} T+\frac{w}{C \rho}
$$

where $T$-temperature; $\tau$-time; $\tau_{r}$-relaxation time; $\alpha$-diffusivity; $w$-volumic power of heat sources; $C$-heat capacity; $\rho$-density. Heat velocity $V_{h}$ is given with the expression $V_{h}=\sqrt{K / \bar{C} \rho \tau_{r}}$, where $K$-thermal conductivity. For example, in aluminium $\tau_{r} \approx 10^{-11} s$ and $V_{h} \approx 3 \mathrm{~km} / \mathrm{s}$.

Hyperbolic equation is valid if material thickness is less than critical value $\left(L_{c r}\right)[3]$ :

$$
L_{c r} \leq 2 \pi \alpha / V_{h}
$$

It has been reported that for metals $L_{c r} \approx 10^{-6} \mathrm{~m}$, for ceramics $L_{c r} \approx 10^{-3} \div 10^{-4} \mathrm{~m}$ [3].

Relevance of these research to TNDT still remains hypothetical. Below we shall deal with parabolic equation and adiabatic problems where heat transfer velocity is determined only by diffusivity parameter. 


\section{http://dx.doi.org/10.21611/qirt.1994.008}

\section{Fast thermal processes (parabolic equation of heat conduction)}

If finite value of heat velocity could be neglected, the Eq.(1) will reduce to common parabolic equation of heat conduction (member $\tau_{r}\left(\partial^{2} T / \partial \tau^{2}\right) \rightarrow 0$ in Eq:(1) ).

\subsection{Basic expressions for pulse heating of uniform solids}

Thermal processes in metals and thin non-metals are adiabatic (especially for short observation times). Theoretical models for one-side TNDT often involve known solutions for semi-infinite body heated with Dirac pulse:

$$
\begin{aligned}
& T(z, \tau)=\frac{W \alpha}{K z} \frac{1}{\sqrt{\pi F o_{z}}} \exp \left(-\frac{1}{4 F o_{z}}\right) ; \quad F o_{z}=\frac{\alpha \tau}{z^{2}} \\
& T(z=0, \tau)=\frac{W}{e \sqrt{\pi \tau}} ; \quad e=\sqrt{K C \rho}
\end{aligned}
$$

If useful information about specimen structure is "hidden" within heating period, the Eqs. $(3,4)$ must be replaced by those including finite duration of heat pulse. For semi-infinite body the corresponding adiabatic solution is as follows:

$$
\begin{aligned}
& T(z, \tau)=\frac{Q z}{k}\left\{2 \sqrt{\frac{F O_{z}}{\pi}} \exp -\frac{1}{4 F o_{z}}-\sqrt{\frac{1}{\pi}}\right) \exp -\frac{1}{4\left(F o_{\bar{z}}-F o_{z_{h}}\right)}- \\
& \left.-\operatorname{erfc}\left(\frac{1}{2 \sqrt{F o_{z}}}\right)\right\} ; \quad F o_{z h}=\frac{\alpha \tau_{h}}{z^{2}} \\
& T(z=0, \tau)=\frac{Q}{k}\left\{2 \sqrt{\frac{\alpha \tau}{\pi}}-2 \sqrt{\frac{\alpha\left(\tau-\tau_{o_{z}}\right)}{\pi}}\right\}
\end{aligned}
$$

Thin or high-conductive specimens are to be modelled with solutions for a plate: .

for Dirac pulse (pulse duration is neglected)

$$
\Theta_{b}=\frac{T(\tau, z=0)}{W \alpha / K L}=1+2 \sum_{n=1}^{\infty} \exp \left(-n^{2} \pi^{2} F_{o}\right) ; \quad F_{o}=\frac{\alpha \tau}{L^{2}}
$$

within square pulse

$$
\Theta_{S P}=\frac{T(\tau, z=0)}{Q L / K}=F o+\frac{1}{3}-\frac{2}{\pi^{2}} \sum_{n=1}^{\infty} \frac{1}{n^{2}} \exp \left(-n^{2} \pi^{2} F o\right)
$$

after square pulse of duration $\tau_{h}\left(F o_{h}\right)$

$$
\Theta_{S P}=\frac{T(\tau, z=0)}{Q L / K}=F o_{h}+\frac{2}{\pi^{2}} \sum_{n=1}^{\infty} \frac{1}{n^{2}} \exp \left(-n^{2} \pi^{2} F o\right)\left[\exp \left(n^{2} \pi^{2} F o_{h}\right)-1\right]
$$

Eq.(7) is more simple but valid for times $\tau>>\tau_{h}$ not providing temperature at the end of heating. Below we shall use Eqs. $(8 a, b)$ which allow to analyse the whole thermal process.

Correlation between models described with Eqs. $(1-8)$ is illustrated with figure 1. It is seen that discrepancy between semi-infinite body and plate for steel becomes noticeable after $120 \mathrm{~ms} \mathrm{(2}$ $\mathrm{mm}$ steel) and after $230 \mathrm{~ms}$ ( $3 \mathrm{~mm}$ steel). Difference between Dirac- and square-pulse model vanishes for steel after about $50 \mathrm{~ms}$. Thinner or/and higher-conductive materials are characterised with shorter transient period and relatively long quasi steady-state period where tomperature is close to $T_{\infty}=W \alpha / K L=Q L F o_{h} / K$ value. 
http://dx.doi.org/10.21611/qirt.1994.008

\section{$\mathrm{T},{ }^{\circ} \mathrm{C}$}

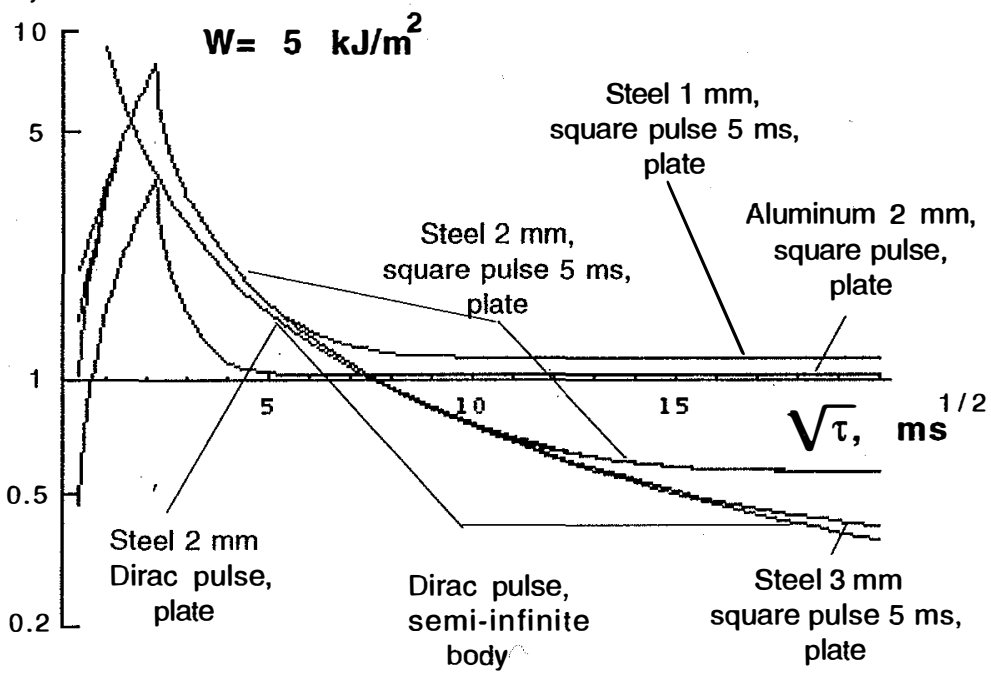

Fig.1. Pulse heating of metals

Time evolution of surface temperature during step-function heating described with Eq.(8a) (i.e. within square pulse) is shown in figure 2 in Log-Log scale for the range of Fourier number from $10^{-4}$ to $10^{1}$ that corresponds to thermal processes in plates with thickness $0.2-5 \mathrm{~mm}$ heated with 1-10 ms heat pulses. Thermal properties range from steel $(K=32 \mathrm{~W} / \mathrm{m} \cdot \mathrm{K}$; $\left.\alpha=7.32 \cdot 10^{-6} \mathrm{~m}^{2} / \mathrm{s}\right)$ to pure graphite $\left(K=174 \mathrm{~W} / \mathrm{m} \cdot \mathrm{K} ; \alpha=153.0 \cdot 10^{-6} \mathrm{~m}^{2} / \mathrm{s}\right)$. In the whole range $F o=10^{-4} \div 10^{1}$ the corresponding function could be approximated with following polynom (average accuracy is about $5 \%$ ):

$$
\begin{aligned}
& Y=0.1531+0.7203 X+0.1150 X^{2}+0.0167 X^{3} \\
& Y=\log \left(\frac{T}{Q L / K}\right) ; \quad X=\log (F o)
\end{aligned}
$$

In the more narrow range $F o=10^{-4} \div 10^{-1}$ the dependence $Y(X)$ could be described with linear function providing accuracy better than $0.1 \%$ :

$$
Y=0.0525+0.5 X
$$

\subsection{Maximum temperature of heating}

At the end of adiabatic heating the temperature is going to $T_{\infty}=Q L F o_{h} / K$ value. Maximum temperature $T_{\max }$ occurs at the end of heat pulse. Hence, the whole process could be characterised with two parameters: $T_{\max }$ and $m_{\max }=T_{\max } / T_{\infty}$ (see Table 1). We have found that these parameters are connected via the following simple formula:

$$
m_{\text {max }}=\frac{1.118}{\sqrt{F o_{h}}}
$$


http://dx.doi.org/10.21611/qirt.1994.008

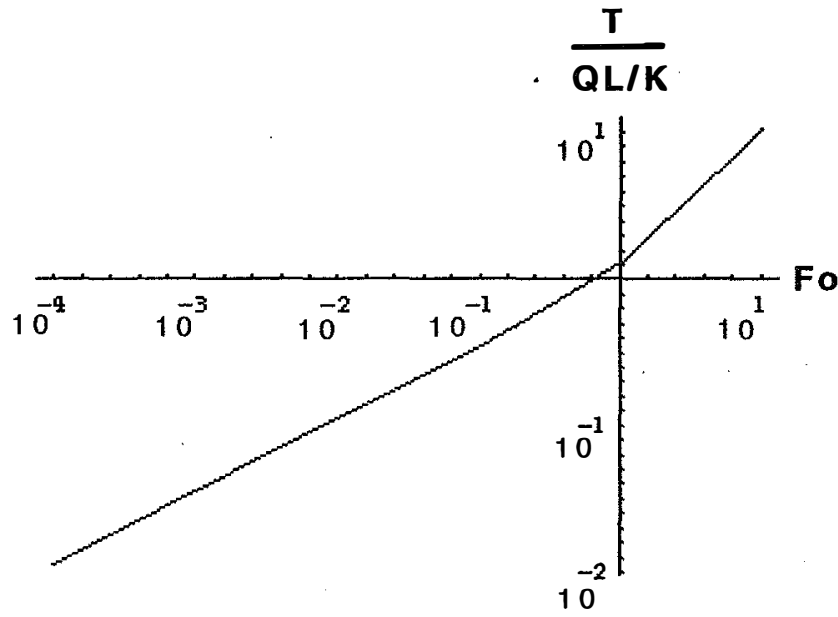

Fig.2. Temperature evolution on a plate surface
within square heat pulse

Table 1 Maximum temperature at the end of square-pulse heating and ratio between maximum and minimum temperatures

\begin{tabular}{|ccc|}
\hline $\begin{array}{l}\text { Dimensionless } \\
\text { heat pulse }\end{array}$ & $\begin{array}{l}\text { Maximum temperature } \\
\text { at the end of heating }\end{array}$ & $\begin{array}{c}\text { Ratio } \\
m_{\max }=T_{\max } / T_{\infty}\end{array}$ \\
\hline duration $F o_{h}$ & $T_{\max }\left(F O_{h}\right) / \frac{Q L}{K}$ & \\
\hline 0.001 & 0.0353 & 35.2776 \\
0.005 & 0.0794 & 15.8770 \\
0.010 & 0.1124 & 11.2433 \\
0.050 & 0.2519 & 5.0382 \\
0.100 & 0.3564 & 3.5642 \\
\hline
\end{tabular}

\subsection{End time of thermal process}

In adiabatic model there is the particular time when difference between surface temperature and value $T_{\infty}=Q L F o_{h} / K$ becomes less than any pre-determined small value $\delta$. It is natural to determine the end time of process $F o_{\text {end }}$ via the following transcendent equation: $\delta=\left[T\left(F o_{\text {end }}\right)-T_{\infty}\right] / T_{\infty}$. For $F O_{h}=0.001-0.1$ and $\delta=0.01-0.1$ the values of $F o_{e n d}$ vary from 0.96 to 1.54 .

\subsection{Fast thermal processes in multilayer structures}

Multilayer

(exception could be made for analysis in Laplace domain [4]). Some of TNDT models use thermal wave conception (e.g., see the work [5]). Here we confine ourselves with remark that idea to measure properties of deeper layers is based on recording the deviations in temperature evolution curve regarding reference curve. It is often stated that each particular layer makes its own contribution to "abnormal" behavior of an evolution curve and the goal of an researcher is 
just to decipher this "hidden" information. According to our experience such a treatment could be easily applied only to the first layer (e.g., coating on a substrate).

Another recent idea in defect characterisation is to apply the so called "early detection" method which requires even shorter observation time than commonly used but could ensure better reproducing a defect shape and size due to weaker 3D heat diffusion [6].

\section{Influence of heat pulse shape}

Shape of heat pulse produced by two "Bowens-3000GHF" tubes (total energy $3 \mathrm{KJ}$ ) is shown in figure $3 a$ for $3 \mathrm{~mm}$ steel specimen. We have approximated the real pulse with $2 \mathrm{~ms}$ square pulse of the same energy. Accuracy in calculating surface temperature, defined as

$\left(T_{\text {real }}-T_{s q}\right) / T_{\text {real }}$, is shown in figure $3 b$. It is seen that equivalent square pulse produces higher temperature in the very beginning of process. Then gradually the contribution of real pulse tail becomes more essential. At $30 \mathrm{~ms}$ the approximation error is less than $4 \%$. Hence, the influence of heat pulse shape must be taken into account for observation time less than $30 \mathrm{~ms}$.

\section{Short heat pulse and IR imager frame time}

One of difficult-to-handle problems in using flash tubes in the TNDT is uneven heating. In solids with uniform surface this difficulty could be overcome by using the normalised contrast $C_{\text {norm }}=T_{d}(\tau) / T_{d}^{\max }-T_{n d}(\tau) / T_{n d}^{\max }$, where "d" and "nd" specify defect and non-defect area. Here we specify another kind of non-uniformity which occurs for shorter observation time (mostly in the first grabbed IR image). There is simulated IR image of non-defect specimen heated for $5 \mathrm{~ms}$ (frame time $40 \mathrm{~ms}$ ) in figure 4 . Specific temperature increase in the middle of image highlights the problem which would arise if small defect is to be found in the first IR image.

Experimental illustration of this effect is shown in figure $A$ (in colour) which presents appearance of the first two IR images in the sequence recorded in flash inspection of $1.8 \mathrm{~mm}$ steel specimen. Temperature was recorded with AGEMA-900 IR imager (frame time in this case was $33 \mathrm{~ms}$ ). Three flash tubes delivered total energy $9 \mathrm{KJ}$ for about $8 \mathrm{~ms}$ (compare tim 3 scale for flash and whole image). The first image shows clearly the flash which "blinds" the camera due to reflected radiation (black paint was used but heat power was obviously too high). This image prompts the problem which could arise if optimum observation time will be within the first $33 \mathrm{~ms}$, e.g. when inspecting aluminium. Notice also quick temperature decrease immediately after flash. The second image reveals two round-shapeof $33 \%$ corrosion areas appearing on the background of overall temperature gradient which occurs as the interaction between cooling process and finite scan speed of the IR camera.

In the conclusion it is worth saying that this effect could be overcome if to use focal plane array (FPA) IR imagers where all pixels are exposed to irradiation simultaneously.

\section{REFERENCES}

[1] VON GUTFELD (R.J.).-Heat pulse transmission. In: Physical Acoustics Principles and Methods, ed. by W.P.Mason, vol.5, 1968, Pergamon Press, N.Y. and London, pp.233-291.

[2] WOLFE (J.P.).-Current topics in phonon imaging. In: Proc. 3rd Int. Conf. Phonon Physics "PHONONS-89", 21-25 August,1989, Heidelberg, Germany, Vol.2, pp.1335-1342.

[3] TIVANOV (G.G).-Analysis of mathematical models used in thermal NDT of composites. Sov.J.NDT, 1987, No. 5, pp.83-85.

[4] MAILLET (D.), HOULBERT (A.S.), DIDIERJEAN (S.) et al. Nondestructive thermal evaluation of delaminations in a laminate: Part I-Identification by measurement of thermal contrast. Composites Sci. and Techn., 1993, Vol.47, pp.137-153.

[5] AAMODT (L.S.), MACLACHLAN SPICER (J.W.), MURPHY (J.S.). Analysis of characteristic heat transit times for time-resolved infrared radiometry studies of multilayered coatings. J. Appl. Physics, Vol.68 (12), 15 December, 1990, pp.6087-6098.

[6] KRAPEZ (J.C.), BALAGEAS (D.), DEOM (A.) and LEPOUTRE (F). - Early Detection by Stimulated Infrared Thermography. Comparison with Ultrasonics and Holo/Shearography. In: "Advances in Signal Processing for Non Destructive Evaluation of Materials", ed. by X. Maldague, NATO ASI Series, Series E: Applied Sciences, Kluwer Academic Publishers, Vol.262, pp.303-321. 
http://dx.doi.org/10.21611/qirt.1994.008

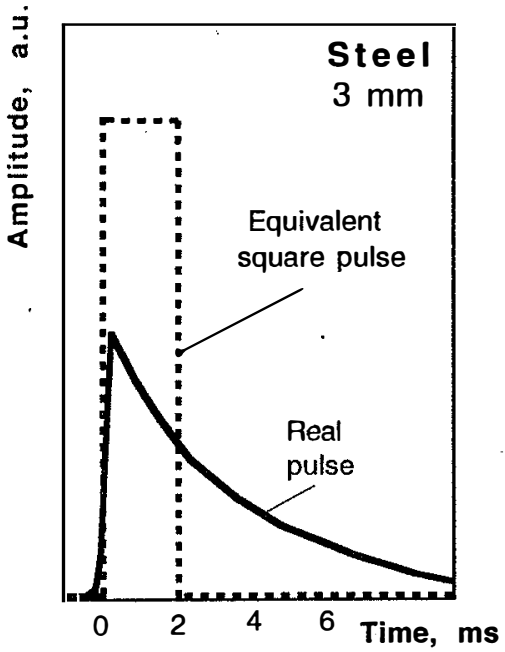

a)

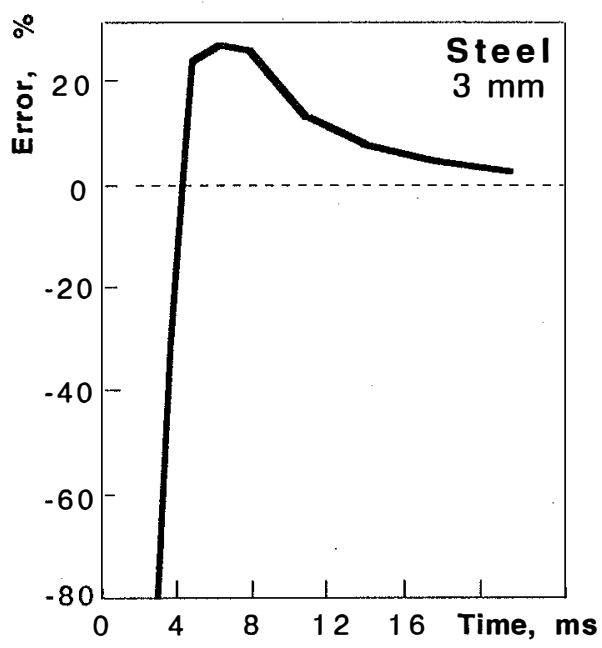

b)

Fig.3. Influence of pulse shape on the results of temperature calculations:

a-tube heat pulse and its approximation, b-calculation error

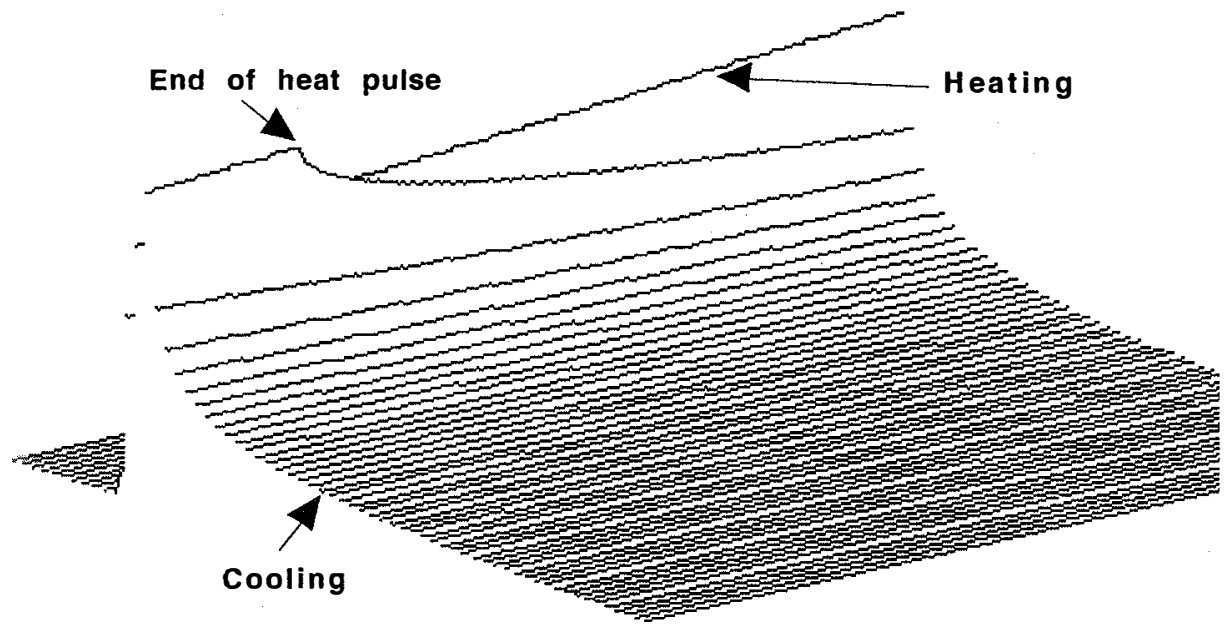

Fig.4. IR image of a specimen uniformly heated with square pulse (heat pulse duration $5 \mathrm{~ms}$, frame time $\mathbf{4 0} \mathrm{ms}$ ) 